\title{
Top Mass and Decay Properties
}

\author{
Jeannine Wagner \\ on behalf of the CDF and D $\varnothing$ Collaborations \\ Universität Karlsruhe - Institut für Experimentelle Kernphysik \\ Wolfgang-Gaede-Str. 1, 76131 Karlsruhe - Germany
}

\begin{abstract}
A summary of the most precise top mass measurements of the D $\varnothing$ and CDF collaborations is presented. The measurements utilize top anti-top candidates in three different decay channels. In addition this article shows most recent measurements of the $W$ helicity in top decay. The integrated luminosity used for all measurements presented in this article ranges between $0.9 \mathrm{fb}^{-1}$ and $1.0 \mathrm{fb}^{-1}$.
\end{abstract}

\section{Introduction}

In 1995 the top quark was discovered at the Tevatron proton-antiproton collider at Fermilab by the CDF and D $\varnothing$ collaborations $[2,3]$. It is the most massive known elementary particle and the Tevatron collider is so far the only place to study the top quark.

At the Tevatron collider, with a center-of-mass energy of $\sqrt{s}=1.96 \mathrm{TeV}$, most top quarks are pair-produced via the strong interaction. In the standard model the top quark decays predominantly into a $W$ boson and a $b$ quark, with a branching ratio close to $100 \%$. Different decay channels are distinguished according to the decay mode of the $W$-boson. Because of the difficult identification of $\tau$-leptons all analyses presented in this article make no use of $\tau$-leptons.

\section{Top Mass Measurements}

A precisely measured top mass $m_{t}$ allows together with the $W$ boson mass for predictions of the Higgs mass. Loops including a top quark lead to a correction of the $\mathrm{W}$ mass which is proportional to $m_{t}^{2}$, while loops including the Higgs lead to a logarithmic correction factor for the $W$ mass. Thus a measurement of the top and $W$ mass leads together with these loop calculations to a constraint on the Higgs mass.

At the Tevatron two analysis techniques are used to measure the top mass. One method is called template method. Here one observable strongly correlated to the true top mass is calculated for each event and the true top mass is then extracted by comparing simulated distributions for signal and background to the data distribution. In the second technique a probability as a function of the true top mass $M_{t}$ and the reconstructed quantities $x$ is calculated for each event separately for $t \bar{t}$ signal and background. Because in the computation of these probabilities enter the leading order matrix element of either signal or background processes this technique is called matrix element method. The total probability $P_{\text {evt }}\left(x \mid M_{t}\right)$ for an event is then obtained by $P_{e v t}\left(x ; M_{t}\right)=f_{\text {top }} \cdot P_{s i g}\left(x ; M_{t}\right)+\left(1-f_{t o p}\right) \cdot P_{b g}(x)$, where the fraction $f_{\text {top }}$ of signal events enters. Then the likelihoods for each event are multiplied and from the sample likelihood the most likely top mass $m_{t}$ is determined.

Much effort has already been put into the measurement of the top mass and meanwhile the uncertainties of the top mass are dominated by systematic uncertainties. The dominating systematic uncertainty is the uncertainty on the jet energy scale. The jet energy scale is a factor applied to the calorimeter jets to get parton level jets. New sophisticated analysis 
strategies had to be developed to make significant progress in the reduction of the top mass uncertainty.

The challenges of the three decay channels and the most precise top mass measurements in each decay channel are presented in the following.

\subsection{Top Mass in the Lepton+Jets Channel}

In the Lepton+Jets decay channel, one top quark decays semileptonically and the second top quark decays hadronically, leading to a signature of one charged lepton, missing transverse energy resulting from the undetected neutrino, and four jets. Out of these four jets both collaborations require at least one jet to be tagged as $b$-jet. The amount of background is at medium level and is composed of mainly $W$-boson events with associated jets and a smaller fraction of QCD multi-jet events. A bonus of this decay channel is the possibility of an in-situ calibration of light quark jets from the hadronically decaying $\mathrm{W}$ boson. This in-situ technique is crucial for the current top mass precision.

The most precise top mass measurements of CDF and D $\varnothing$ both make use of the in-situ technique, leading to a strong reduction of the dominant systematic uncertainty, the jet energy scale. Both measurements use matrix element methods. The CDF measurement [4] yields $m_{t}=170.8 \pm 2.2$ (stat.) \pm 1.4 (syst.) $\mathrm{GeV} / \mathrm{c}^{2}$ and the $\mathrm{D} \varnothing$ measurement [5] yields $m_{t}=170.5 \pm 2.4$ (stat.) \pm 1.2 (syst.) $\mathrm{GeV} / \mathrm{c}^{2}$. Both measurements are well consistent with each other and very competitive. In case of the CDF measurement the largest uncertainty comes now from the initial and final state radiation, the second largest uncertainty is due to the $b$-jet energy scale uncertainty, while in case of the $\mathrm{D} \varnothing$ measurement the largest uncertainty is due to the $b$-jet energy scale uncertainty.

\subsection{Top Mass in the Dilepton Channel}

In the Dilepton decay channel, both top quarks decay semileptonically, leading to a signature of two charged leptons, large missing transverse energy resulting from the two undetected neutrinos, and two jets. The amount of background events is at low level, where mainly di-boson events and events with a real $W$ - or $Z$-boson and associated jets contribute. The challenge of this decay channel is the under-constrained system due to the presence of two neutrinos.

The most precise CDF measurement [6] is a matrix element method. In order to deal with the under-constrained system the event probabilities $P_{\text {evt }}$ are integrated over both unmeasured neutrino energies. The measurement yields $m_{t}=164.5 \pm 3.9$ (stat.) \pm 3.9 (syst.) $\mathrm{GeV} / \mathrm{c}^{2}$.

The most precise measurement of $\mathrm{D} \varnothing[7]$ is a template method. In order to deal with the under-constrained system, the pseudorapidities of the two neutrinos are assumed for a hypothetical top mass. For each hypothesis an expected missing transverse momentum is calculated and a weight according to the agreement between the calculated and the observed missing transverse energy is then assigned to this hypothesis. For each event the mean and root mean square (rms) values of the distribution: weights versus hypothetical top mass, are determined and used to fit the top mass. The result of this measurement is $m_{t}=172.5 \pm 5.8$ (stat.) \pm 5.5 (syst.) $\mathrm{GeV} / \mathrm{c}^{2}$. 


\subsection{Top Mass in the All Hadronic Channel}

In the All Hadronic decay channel, both top quarks decay hadronically, leading to a signature of six jets. Out of these six jets at least one jet is required to be tagged as $b$-jet. The amount of background events is huge and consists mainly of multi-jet events. The actual challenge of this channel is the reduction of the huge background. That is the reason why a further selection on the event topology is necessary, for example with a neural net. A bonus of this channel is again the possibility of an in-situ calibration of light quark jets using the mass of the hadronically decaying $W$ bosons.

So far, only CDF has performed measurements in this decay channel. The most precise measurement [8] applies a matrix element assisted 2D template method. It uses for the first time the in-situ technique. The signal templates are determined from matrix element calculations, while the background templates are determined from a data-driven model. The top mass is then determined from a fit of the signal and background templates to the data. This analysis yields a top mass of $m_{t}=171.1 \pm 3.7$ (stat.) \pm 2.1 (syst.) $\mathrm{GeV} / \mathrm{c}^{2}$.

\subsection{Tevatron Top Mass Combination}

A summary of the most precise top mass measurements is presented in Figure 1. The Tevatron top mass combination [9]

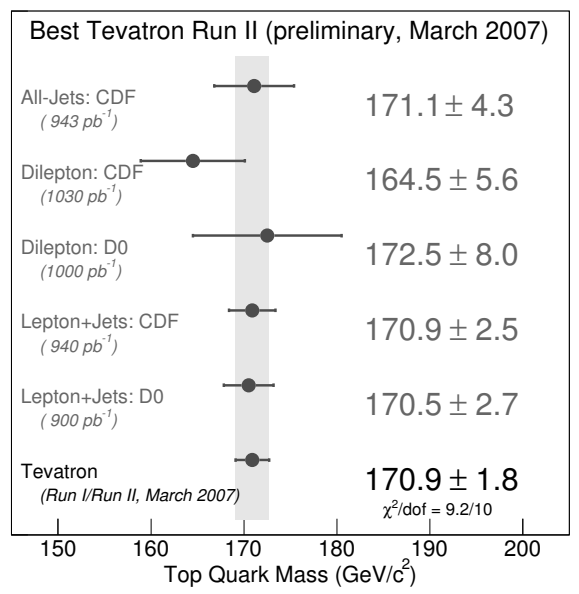

Figure 1: Most precise top mass measurements and Tevatron top mass combination. including all these new very precise top mass measurement yields $m_{t}=170.9 \pm$ 1.8(stat.+syst.) $\mathrm{GeV} / \mathrm{c}^{2}$, corresponding to a relative uncertainty of the top mass below $1.1 \%$.

\section{Top Decay Properties}

Although the top mass is now measured with a relative uncertainty at the $1 \%$ level the question remains whether the standard model successfully predicts the properties of the top quark. This article addresses one interesting aspect of top quark decay properties, namely the $W$ helicity in top decays.

In general the $\mathrm{W}$ boson can either be longitudinally, left-handed or right-handed polarized. In the standard model a fraction $F_{0}$ of longitudinally polarized $\mathrm{W}$ bosons of $70 \%$ is predicted, the fraction $F_{-}$of left-handed $\mathrm{W}$ bosons is predicted to be $30 \%$ while the fraction $F_{+}$of right-handed $\mathrm{W}$ boson is in the SM strongly $\mathrm{V}-\mathrm{A}$ suppressed and is predicted to be zero. Deviations from these $\mathrm{SM}$ values would indicate new physics. A possible $\mathrm{V}+\mathrm{A}$ coupling would lead to an altered $F+$ value. An altered $F_{0}$ value would be an indication for an non-SM electroweak symmetry breaking mechanism. 
All measurements look at the leptonic decay of the $\mathrm{W}$ boson from the top quark. In the most recent measurements $\cos \theta^{*}$ is used as observable, with $\theta^{*}$ being the angle between the charged lepton and the negative direction of the top quark in the rest frame of the $W$-boson.

The two most recent $W$ helicity results are performed by CDF [10] and they both utilize the Lepton+Jets channel. Performing a fit to the distribution of the reconstructed $\cos \theta^{*}$, the fit yields $F+=$ $-0.03 \pm 0.07$ when $F_{0}$ is fixed to its Standard Model value of 0.7 , while the fit yields

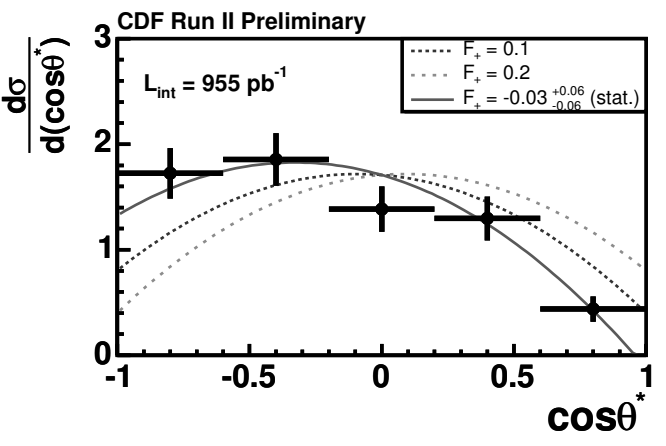
$F_{0}=0.59_{-0.13}^{+0.14}$ if $F_{+}$is fixed to the Standard

Figure 2: Unfolded differential $\cos \theta^{*}$ distribution.

Model value of 0 . Figure 2 shows the unfolded differential $\cos \theta^{*}$ distribution. In a second analysis a simultaneous fit of $F_{0}$ and $F_{+}$has been performed for the first time, yielding: $F_{+}=-0.06 \pm 0.10$ and $F_{0}=0.74 \pm 0.26$. However, the uncertainties are still large for the simultaneous fit and even in the constrained fit the result is still statistically limited. The $F_{+}$measurement from CDF using the invariant mass of the charged lepton and the $b$-jet [11] and the $F_{+}$measurement from $\mathrm{D} \varnothing[12]$ give consistent results. In summary, all $\mathrm{D} \varnothing$ and CDF measurements are consistent with the Standard Model prediction.

\section{Summary}

In this article recent measurements of the top mass and of top decay properties have been presented. By now the top mass measurements are systematically limited. The new Tevatron top mass combination yields $m_{t}=170.9 \pm 1.8 \mathrm{GeV} / \mathrm{c}^{2}$ leading to a relative uncertainty at the $1 \%$ level. Concerning the top decay properties, all measurements are still statistically limited and so far no deviation compared to the Standard Model prediction is observed.

\section{References}

[1] Slides: http://indico. cern. ch/contributionDisplay. py?contribId=107\&sessionId=9\&conf Id=9499

[2] F. Abe et al., Phys. Rev. Lett. 742626 (1995).

[3] S. Abachi et al., Phys. Rev. Lett. 742632 (1995).

[4] A. Abulencia et al., FERMILAB-PUB-07-070-E, submitted to Phys. Rev. Lett..

[5] DØ conference note 5362 (2007).

[6] A. Abulencia et al., Phys. Rev. D75, 031105(R) (2007).

[7] DØ conference note 5347 (2007).

[8] A. Abulencia et al., Phys. Rev. Lett. 98, 142002 (2007).

[9] Tevatron Electroweak Working Group, arXiv:hep-ex/0703034 (2007).

[10] CDF conference note 8380 (2006); CDF conference note 8368 (2006).

[11] A. Abulencia et al., Phys. Rev. Lett. 98, 072001 (2007).

[12] V. M. Abazov et al., Phys. Rev. D75, 031102(R) (2007). 